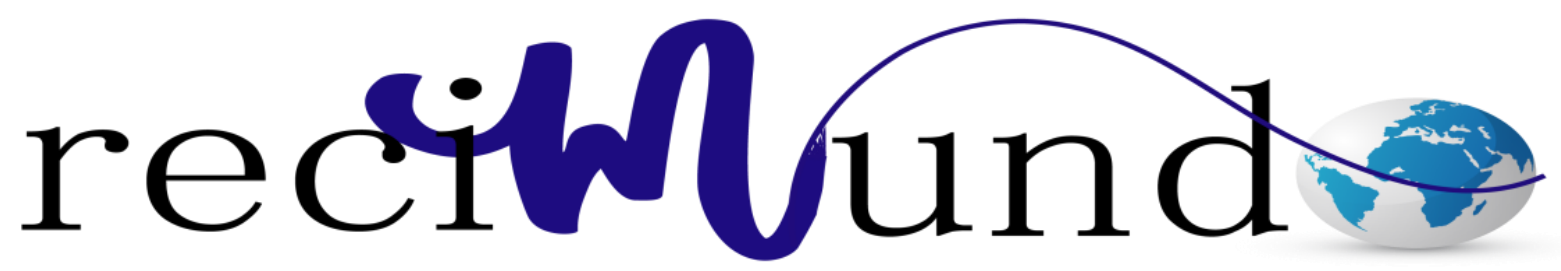

Revista Científica Mundo de la Investigación y el Conocimiento

Mariela Gissela Macías Intriago a; Ivonne Jasmina Tacoamán Acurio ${ }^{\text {b; }}$ Luis Alberto Giler Saltos '; William Mauricio Quimis Guerrido ${ }^{\text {d; }}$ María Lourdes Choez Reyes ${ }^{\mathrm{e}}$; Melissa Mercedes Álvarez Lara ${ }^{\mathrm{f}}$

Parto humanizado como estrategia de salud pública en atención primaria de salud Humanized partition as a public health strategy in primary health care

Revista Científica Mundo de la Investigación y el Conocimiento. Vol. 2 núm.3, septiembre, ISSN: 2588-073X, 2018, pp. 730-745

DOI: 10.26820/recimundo/2.(3).septiembre.2018.730-745

Editorial Saberes del Conocimiento

Recibido: 05/04/2018 Aceptado: 15/05/2018_ Publicado: 28/08/2018

Correspondencia: 1ags-89@hotmail.com

a. Doctor en Medicina y Cirugía, Mg en Epidemiologia; Directora Distrital de Salud 13D01 Portoviejo; Docente de la Facultad de Ciencias de la Salud - Universidad Técnica de Manabí.

b. Obstetriz; Magister en Gerencia en Salud para el Desarrollo Local; Centro de Salud Las Cumbres; Servidor Público 7.

c. Médico Residente; Hospital IESS de Portoviejo; lags-89@hotmail.com

d. Distrito 13D03 Jipijapa Puerto López Salud; Medico General Asistencial.

e. Obstetra; Hospital Básico Israel Quintero Paredes.

f. Enfermera; Analista de Promoción de Salud e igualdad del Distrito 13D03 Jipijapa Puerto López Salud. 


\section{Parto humanizado como estrategia de salud pública en atención primaria de \\ salud}

Vol. 2, núm. 3., (2018)

Mariela Gissela Macías Intriago; Ivonne Jasmina Tacoamán Acurio; Luis Alberto Giler Saltos; William Mauricio Quimis Guerrido; María Lourdes Choez Reyes; Melissa Mercedes Álvarez

Lara

\section{RESUMEN}

El parto con pertinencia intercultural, se inscribe dentro de la política del Ministerio de Salud Pública que propone la atención integral de salud tanto a la madre como al niño. Se realizó una investigación desde las perspectivas de los investigadores para analizar la estrategia del ente rector en salud en la atención primaria y su contribución a la incidencia en la seguridad de la madre y la reducción de complicaciones obstétricas, con potencialidad para incidir en los profesionales de la salud, el alcance del mismo preciso una revisión documental. El autor concluyó que el parto humanizado ofrece a toda mujer la opción de practicar tradicional y libremente la posición del parto dentro de un ambiente seguro donde en caso de una emergencia obstétrica existen las condiciones necesarias para evitar secuelas, muertes maternas y neonatales. Los profesionales de la salud pueden encontrar en esta estrategia acciones adecuadas de salud para la atención del parto institucional en el siglo XXI, así como también para la reducción del riesgo de mortalidad materna y neonatal.

Palabras claves: Parto humanizado, salud, atención primaria, estrategia, intercultural. 


\title{
Parto humanizado como estrategia de salud pública en atención primaria de salud
}

Vol. 2, núm. 3., (2018)

Mariela Gissela Macías Intriago; Ivonne Jasmina Tacoamán Acurio; Luis Alberto Giler Saltos;

William Mauricio Quimis Guerrido; María Lourdes Choez Reyes; Melissa Mercedes Álvarez Lara

\begin{abstract}
Childbirth with intercultural relevance, is part of the policy of the Ministry of Public Health that proposes comprehensive health care to both mother and child. Research was carried out from the perspectives of the researchers to analyze the strategy of the governing body in health in primary care and its contribution to the incidence in the safety of the mother and the reduction of obstetric complications, with the potential to affect professionals in health, the scope of the same precise a documentary review. The author concluded that humanized childbirth offers every woman the option of traditionally and freely practicing the position of childbirth within a safe environment where, in the case of an obstetric emergency, there are the necessary conditions to avoid sequelae, maternal and neonatal deaths. Health professionals can find in this strategy appropriate health actions for institutional birth care in the 21 st century, as well as for reducing the risk of maternal and neonatal mortality.
\end{abstract}

Keywords: Humanized childbirth, health, primary care, strategy, intercultural. 


\section{Parto humanizado como estrategia de salud pública en atención primaria de \\ salud}

Vol. 2, núm. 3., (2018)

Mariela Gissela Macías Intriago; Ivonne Jasmina Tacoamán Acurio; Luis Alberto Giler Saltos; William Mauricio Quimis Guerrido; María Lourdes Choez Reyes; Melissa Mercedes Álvarez

Lara

\section{Introducción.}

En la actualidad existe una tendencia hacia un cambio que establezca un nuevo marco conceptual en la asistencia sanitaria perinatal en la que se pretende dar una atención más humana y cercana, donde la mujer sea la protagonista de su propio embarazo y parto dejando atrás un modelo medicalizado (Ministerio de Sanidad y Consumo, 2007).

El concepto de parto humanizado, ha sido publicado por muchos autores desde que la Organización Mundial de la Salud (1985) alertara sobre el elevado grado de medicalización en la atención al parto y desarrollara las recomendaciones fundamentales para llevar a cabo un modelo humanizado de atención al parto (García, Guillén, \& Acevedo, 2010).

La creación y publicación de este tipo de estrategias que buscan incorporar a la práctica clínica un cambio hacia la humanización del parto, no es exclusiva de España, sino que son muchos los países que pretenden iniciar este cambio como Brasil o Japón (Martínez G. J., 2011). Y a raíz de (1996), con la publicación de la guía "Cuidados en el parto normal: una guía práctica" elaborado por la OMS en Ginebra surgen varios documentos que abogan por un parto normal.

El término Humanización del parto, se contempla por primera vez en Ceará (Ministerio de Salud de Brasil, 2000), recogido en el Programa de Humanización en el Prenatal y Nacimiento, el cual fue instituido con base en los análisis de las necesidades de atención específica a la gestante, al recién nacido y a la madre en el periodo posparto. El objetivo primordial del Programa de Humanización en el Prenatal y Nacimiento (PHPN) es asegurar la 


\section{Parto humanizado como estrategia de salud pública en atención primaria de salud}

Vol. 2, núm. 3., (2018)

Mariela Gissela Macías Intriago; Ivonne Jasmina Tacoamán Acurio; Luis Alberto Giler Saltos; William Mauricio Quimis Guerrido; María Lourdes Choez Reyes; Melissa Mercedes Álvarez Lara

mejora del acceso, de la cobertura y de la calidad del acompañamiento prenatal, de la asistencia al parto y puerperio a las gestantes y al recién nacido, en la perspectiva de los derechos de la ciudadanía. En la Declaración de Ceará en torno a la Humanización se pone de manifiesto la trascendental importancia de esta nueva visión del parto: "El parto y el nacimiento son el principio y punto de partida de la vida y son, por ello, procesos que afectan al resto de la existencia humana. De ahí que la humanización del parto constituya una necesidad urgente y evidente. Por tanto, creemos firmemente que la aplicación de la humanización en los cuidados que se proveen al comienzo de la vida humana será determinante y definitiva para las sociedades futuras".

La humanización comprende por lo menos dos aspectos fundamentales. El primero, respecto a la convicción que es deber de las unidades de salud recibir con dignidad la mujer, sus familiares y el recién nacido, es decir, acogerlas y tratarlas como personas. Esto requiere actitud ética y solidaria por parte de los/as profesionales de la salud y la organización de la institución con la finalidad de crear un clima agradable y a instituir rutinas hospitalarias que rompan con el tradicional aislamiento impuesto a la mujer (Iglesias \& Alli, 2009).

El otro se describe a la adopción de medidas y procedimientos que son beneficiosos para el acompañamiento del parto y del nacimiento, evitando prácticas intervencionistas innecesarias, que aunque tradicionalmente han sido realizadas, no benefician a la mujer ni al recién nacido, y que con frecuencia acarrean mayores riesgos para ambos. Además dignificaría a la mujer pues 


\section{Parto humanizado como estrategia de salud pública en atención primaria de \\ salud}

Vol. 2, núm. 3., (2018)

Mariela Gissela Macías Intriago; Ivonne Jasmina Tacoamán Acurio; Luis Alberto Giler Saltos; William Mauricio Quimis Guerrido; María Lourdes Choez Reyes; Melissa Mercedes Álvarez

Lara

tendría en cuenta sus deseos y necesidades a la hora de parir que contribuiría a aumentar su grado de satisfacción (Lais \& Gilbertg, 2012).

En lo que se refiere al parto natural, no existe organización internacional que lo defina. Para adentrarnos y estudiar el concepto en el posterior análisis de contenido, tomamos como referencia el establecido según "la Asociación el Parto es Nuestro", la cual contempla que "Un parto natural es ante todo un parto aquel que se respetan los tiempos normales de la mujer y el niño que va a nacer. En el que no se realizan intervenciones innecesarias ni se aplican protocolos rutinarios por sistema. De lo que se trata es de llevar a la práctica el avance de la ciencia, los últimos descubrimientos científicos, la medicina basada en la evidencia y ofrecer a la madre la máxima seguridad y el respeto por su cuerpo y el del bebé”. (Rodríguez, 2013).

Humanizar el parto significa poner a la mujer que está dando a luz en el centro y en el control de tal manera que ella y no los médicos o cualquier otra persona tome todas las decisiones de lo que va a ocurrir. Humanizar el parto significa entender que el foco de los servicios maternales es la atención primaria basada en la comunidad, no los hospitales basados en cuidados terciarios con matronas, enfermeras y médicos todos trabajando en armonía como iguales (Marsden, 2016).

En los últimos años se está produciendo un cambio más que evidente en el modelo de atención sanitaria al parto, aumentando la importancia del concepto de humanización relacionado principalmente con la atención sanitaria ofrecida por los profesionales durante el trabajo de parto (Biurrun \& Goberna, 2013). 


\section{Parto humanizado como estrategia de salud pública en atención primaria de salud}

Vol. 2, núm. 3., (2018)

Mariela Gissela Macías Intriago; Ivonne Jasmina Tacoamán Acurio; Luis Alberto Giler Saltos; William Mauricio Quimis Guerrido; María Lourdes Choez Reyes; Melissa Mercedes Álvarez Lara

(Goberna, Banús, Linares, \& Salas, 2008). Debemos destacar que la no tecnificación del parto, por sí sola, no garantiza que la atención al mismo sea humanizada. Para ello, es indispensable que tanto las gestantes como sus parejas tengan la información y el conocimiento necesarios de las posibles opciones disponibles de atención al parto y conozcan los riesgos y beneficios de cada una de ellas.

Es por ello que el Ecuador toma el reto de cumplir con los Objetivos del Milenio; siendo uno de ellos la reducción de la Muerte Materna por lo cual el Ministerio de Salud Pública ha creado nuevas estrategias y normativas para mejorar la atención del parto y puerperio permitiendo que las mujeres se conviertan en las protagonistas de su parto (Secretaría Nacional de Planificación, 2007).

En la provincia de Pichincha ha sido creada una clínica especializada en el parto humanizado y acuático. Se encuentra en Cumbayá a las afueras de Quito, en el sector la primavera. Alarcón (2016), director de la clínica afirma que: El parto humanizado es la mejor forma de que un ser humano llegue a este mundo como esfuerzo del posicionamiento y desarrollo de la salud intercultural, con visión y misión institucional por una parte y por otra como planteamiento inserto en el Plan Nacional de Salud y Derechos Sexuales y Reproductivos y en el Plan de Reducción de la Mortalidad Materna y a la fecha en el Modelo de Atención Integral de Salud con enfoque Intercultural y Comunitario.

¿Puede la estrategia del parto humanizado contribuir a la formación de valores humanos, éticos y morales en los profesionales de la Salud? Al respecto, los autores se propusieron analizar 


\section{Parto humanizado como estrategia de salud pública en atención primaria de \\ salud}

Vol. 2, núm. 3., (2018)

Mariela Gissela Macías Intriago; Ivonne Jasmina Tacoamán Acurio; Luis Alberto Giler Saltos; William Mauricio Quimis Guerrido; María Lourdes Choez Reyes; Melissa Mercedes Álvarez

Lara

la estrategia nacional desde la perspectiva de diferentes investigadores de la historia contemporánea y su contribución en la actividad diaria de los profesionales de salud.

\section{Metodología.}

Se trata de una revisión narrativa-descriptiva, basada en el análisis de la literatura publicada en libros, artículos de revistas y análisis crítico del autor con el objetivo de integrar, describir y discutir el conocimiento existente sobre el parto humanizado.

\section{Desarrollo.}

El Parto culturalmente adecuado ha contribuido a mejorar la calidad de la atención a las pacientes, la relación médico paciente, ha permitido compartir la experiencia del parto con su familia, ha incrementado la asistencia a los controles prenatales y educación prenatal dando como resultado el bienestar del binomio madre- hijo.

El parto y el nacimiento son el principio y punto de partida de la vida y son, por ello, procesos que afectan al resto de la existencia humana. De ahí que la humanización del parto constituya una necesidad urgente y evidente. Por tanto, creemos firmemente que la aplicación de la humanización en los cuidados que se proveen al comienzo de la vida humana será determinante y definitiva para las sociedades futuras (Freyermuth \& Sesia, 2009).

En el estudio realizado en la ciudad de Brasil, se afirma que para poder humanizar un parto es necesario «empoderar» a la mujer, fomentando su participación activa y su toma de decisiones en todos los aspectos de su propio cuidado. Además, los profesionales que atienden el 


\section{Parto humanizado como estrategia de salud pública en atención primaria de salud}

Vol. 2, núm. 3., (2018)

Mariela Gissela Macías Intriago; Ivonne Jasmina Tacoamán Acurio; Luis Alberto Giler Saltos; William Mauricio Quimis Guerrido; María Lourdes Choez Reyes; Melissa Mercedes Álvarez Lara

parto deben trabajar como iguales, usando la tecnología basada en la evidencia y fomentando la atención primaria (Misago \& Kendall, 2001).

En ese mismo año, Wagner (2001) publica uno de los artículos de referencia sobre la atención humanizada al nacimiento, y en el que sostiene que humanizar el parto consiste en entender que la mujer que da a luz es un ser humano, y no una máquina para hacer bebés. Según este autor, el parto humanizado es aquel que sitúa a la mujer en el centro y control del proceso para que sea ella, y no el personal sanitario, quien tome las decisiones acerca de lo que sucede.

Hodnett (2006), señala que la práctica del parto humanizado tiene implicaciones en los aspectos emocionales de la atención a la maternidad, como el apoyo continuo durante el parto y el alumbramiento, y asegura que prestar atención a los aspectos psicológicos puede ser una estrategia más eficaz durante el parto que las intervenciones tecnológicas

En (2010), Díaz-Tello y Paltrow afirman que los términos humanización y parto están muy relacionados y deben incorporarse en el concepto de justicia reproductiva, que no ha de basarse sólo en otorgar poder a la mujer en la toma de decisiones cuando desea tener hijos, sino también en permitirle decidir cómo quiere ser tratada durante el proceso del embarazo y el parto.

Behruzi et al. (2014), aseguran que no existe conflicto entre el nacimiento humanizado y la intervención médica en esa situación, y señalan que entre los obstáculos para la prestación de atención sanitaria en un parto humanizado en un embarazo de alto riesgo figuran factores como la presión de ser responsable de la seguridad de la madre y el feto, la falta de participación activa de la mujer en la toma de decisiones y la pesada carga de responsabilidad sobre los sanitarios por 


\section{Parto humanizado como estrategia de salud pública en atención primaria de \\ salud}

Vol. 2, núm. 3., (2018)

Mariela Gissela Macías Intriago; Ivonne Jasmina Tacoamán Acurio; Luis Alberto Giler Saltos; William Mauricio Quimis Guerrido; María Lourdes Choez Reyes; Melissa Mercedes Álvarez

Lara

los posibles problemas legales. Los factores que facilitan el parto humanizado en un embarazo de alto riesgo incluyen el uso compartido de la toma de decisiones y otras responsabilidades diversas entre los profesionales sanitarios y las mujeres.

El parto humanizado es relevante en todos los países y en todos los ámbitos de parto. Aun si éste requiere de intervenciones o cirugía abdominal, debe realizarse con respeto a los principios del parto humanizado. La incorporación del mismo en los ámbitos de salud pública de un país genera los siguientes resultados (Freyermunth, 2013):

- Menores tasas de mortalidad y morbilidad materno-perinatales.

- Una experiencia del parto cualitativamente más satisfactoria para la mujer y su familia.

- Un importante ahorro económico para el sistema de salud pública.

- Mayores tasas de éxito en la lactancia materna que constituye un factor importante en prevención y promoción de la salud.

Desde el año 2016 se buscó la implementación de las normas que propone la Guía Técnica de Parto Culturalmente adecuado y la estrategia ESAMyN. Canteras, M., Ballesteros, C., Carrillo (2017), mediante un estudio expresan: La humanización del parto que comprende: el deber del personal médico de recibir a la madre, al recién nacido y a sus familiares con dignidad y respeto, así como también, ofrecer a la madre procedimientos que faciliten el acompañamiento del parto y dejen de lado las acciones farmacológicas innecesarias.

La atención sanitaria obstétrica actual contempla la seguridad y la calidad: seguridad para reducir la morbilidad materno-infantil y calidad para ofrecer una atención sanitaria acorde a la 


\section{Parto humanizado como estrategia de salud pública en atención primaria de salud}

Vol. 2, núm. 3., (2018)

Mariela Gissela Macías Intriago; Ivonne Jasmina Tacoamán Acurio; Luis Alberto Giler Saltos; William Mauricio Quimis Guerrido; María Lourdes Choez Reyes; Melissa Mercedes Álvarez Lara

evidencia científica actual y capaz de satisfacer las necesidades y deseos de las familias (Fernández, 2017).

La Atención Primaria supone el primer contacto de la mujer y su pareja con la asistencia sanitaria al conocer su embarazo, y como tal debe ser la responsable de brindar a esta nueva familia una atención prenatal de calidad, la cual nos ofrece la oportunidad de comunicarnos con las mujeres, las familias y las comunidades y ofrecerles apoyo en un momento decisivo en la vida de una mujer (2016).

Martínez y Delgado (2014), indican que la estrategia de humanización de la asistencia perinatal de las administraciones sanitarias, la preparación al nacimiento representa un instrumento fundamental, ya que ha demostrado que reduce el número de intervenciones sistemáticas e innecesarias durante el embarazo y el parto y que existe una asociación negativa con el número de partos que acaban en cesárea en embarazos de bajo riesgo. Además, la asistencia a los cursos de educación maternal incide de manera positiva a la hora de reconocer adecuadamente el momento del parto y acudir al servicio de obstetricia, evitando desplazamientos innecesarios de la mujer y estancias hospitalarias prolongadas.

En las actuales entidades desconcentradas de salud, la cantidad de pacientes y el estrés laboral que viven los profesionales de salud hacen complicado el acompañamiento personalizado para cada mujer. Es así que un estudio realizado en Chile (2018) aborda que "solo el 12.4\% del tiempo de la enfermera se dedica a dar apoyo a la paciente”. Lo que hacen aún más indispensable 


\section{Parto humanizado como estrategia de salud pública en atención primaria de}

salud

Vol. 2, núm. 3., (2018)

Mariela Gissela Macías Intriago; Ivonne Jasmina Tacoamán Acurio; Luis Alberto Giler Saltos; William Mauricio Quimis Guerrido; María Lourdes Choez Reyes; Melissa Mercedes Álvarez

Lara

el apoyo de un familiar en el momento del parto. El parto se medicaliza, lo que significa que, pese a que no existían patologías, la intervención médica estaba presente de manera decisiva.

En varios países ya se han aprobado leyes que protegen el embarazo y al recién nacido. Por ejemplo: Venezuela fue el primer país en utilizar el término "violencia obstétrica en el año 2007 donde fue aprobado la ley más conocida como "Ley de Parto Humanizado", posteriormente fue aprobado en Argentina en el año 2009 y en el año 2014 fue México quien reconoció la necesidad de erradicar ese tipo de violencia.

El parto culturalmente adecuado es una estrategia que nos permite cumplir con los objetivos del Plan Nacional cuyas metas son: disminuir en un 35\% la mortalidad materna y neonatal precoz, y aumentar al 70\% la cobertura de parto institucional (2017). Actualmente se evidencia que existe una gran cantidad de mujeres indígenas y afro ecuatorianas que prefieren dar a luz en los establecimientos creados por el estado y adecuados con el fin de prevenir las complicaciones materno-neonatales.

En España se encuentran realizando un proceso de cambio paradigmático de la asistencia al parto y al nacimiento. Múltiples factores científicos y sociales de diversa naturaleza están impulsando este tránsito del antiguo modelo de atención a uno nuevo, caracterizado por el respeto a la fisiología del parto, al protagonismo de la mujer y al ejercicio de su autonomía, y por una atención sanitaria personalizada y basada en la evidencia científica actualmente disponible (Direccion General de Salud Pública , 2011). Entre estos factores podríamos citar:

- La mayor comprensión de la importancia de atender los aspectos emocionales y 


\section{Parto humanizado como estrategia de salud pública en atención primaria de salud}

Vol. 2, núm. 3., (2018)

Mariela Gissela Macías Intriago; Ivonne Jasmina Tacoamán Acurio; Luis Alberto Giler Saltos; William Mauricio Quimis Guerrido; María Lourdes Choez Reyes; Melissa Mercedes Álvarez Lara

sociales de un acontecimiento de la trascendencia del parto y del nacimiento para mejorar el bienestar de la madre y del recién nacido, favorecer el establecimiento de un vínculo estrecho entre ellos e incrementar la autoconfianza de la madre, tan necesaria para la crianza y el amamantamiento.

- El desarrollo del conocimiento científico de la fisiología del parto y de las necesidades básicas de la madre y el hijo o hija durante el parto y tras el nacimiento.

- El auge de la Medicina basada en la evidencia que está poniendo de manifiesto que el uso inapropiado de la tecnología en el parto, lejos de aumentar la seguridad, ocasiona yatrogenia, incrementa la tasa de cesáreas y partos instrumentales e interfiere en el establecimiento del vínculo materno-filial.

- La preocupación social, expresada en particular por grupos de mujeres organizadas en torno a la defensa de la maternidad consciente, sobre la actual deshumanización de la atención al parto y nacimiento, la injustificada medicalización de los partos normales y el escaso protagonismo de la mujer y su pareja en todo el proceso. Este cambio de paradigma no está resultando fácil. Muchos son los retos que las y los profesionales sanitarios, los servicios de salud, las mujeres y la sociedad en su conjunto deben afrontar para realizarlo.

El Ecuador está comprometido con el cumplimiento de los Objetivos del Milenio Para cumplir con este objetivo y con los compromisos asumidos en la conferencia Internacional de Población y Desarrollo, el país ha desplegado un marco jurídico legal que crea un entorno habilitante para la reducción de la mortalidad materna (2018).

Es así que los Establecimientos de Salud como Amigos de la Madre y del Niño (ESAMyN) (2018), buscan en esta una estrategia implementada con el objetivo de disminuir la mortalidad materna y neonatal". Podemos decir que son las propias mujeres que acuden a dar a luz las que demandan un trato más humano, empatía, más información y participación en los 


\section{Parto humanizado como estrategia de salud pública en atención primaria de}

salud

Vol. 2, núm. 3., (2018)

Mariela Gissela Macías Intriago; Ivonne Jasmina Tacoamán Acurio; Luis Alberto Giler Saltos; William Mauricio Quimis Guerrido; María Lourdes Choez Reyes; Melissa Mercedes Álvarez

Lara

procesos de parto, lo cual requiere de la sensibilización de los profesionales de la salud y del desarrollo de estrategias para el cuidado integral de la mujer en el parto. El cuidado médico, biológico no es el único que debe ser tenido en cuenta, sino también los aspectos culturales, emocionales, psicológicos, sexuales, ambientales, personales.

El papel del Estado es primordial en la constitución y promulgación de leyes y normas referidas a la humanización y respeto en la atención al parto dentro de las instituciones de salud, garantizando que en ellas sean aplicadas, así como también la formación profesional de todos los integrantes de los equipos de salud que interactúan en el medio.

\section{Conflicto de intereses}

Los autores declaran que no existen conflictos de intereses.

\section{Declaración de contribución}

Todos los autores participaron en la búsqueda de información y en la redacción del artículo.

\section{Bibliografía.}

Alarcón, D. (2016, 07 2). Parto humanizado. (N. Viracucha, Interviewer)

Behruzi, R., Hatem, M., Goulet, L., Fraser, W., Leduc, N., \& Misago, C. (2014). Humanized birth in high risk pregnancy: barriers and facilitating factors. Med Heald Care Philos, 4958 .

Biurrun, G. A., \& Goberna, T. J. (2013). La humanización del trabajo de parto: necesidad de definir el concepto. Matronas Prof., 62-66. 


\section{Parto humanizado como estrategia de salud pública en atención primaria de salud}

Vol. 2, núm. 3., (2018)

Mariela Gissela Macías Intriago; Ivonne Jasmina Tacoamán Acurio; Luis Alberto Giler Saltos;

William Mauricio Quimis Guerrido; María Lourdes Choez Reyes; Melissa Mercedes Álvarez Lara

Canteras, M., Ballesteros, C., \& Carrillo, C. (2017). Comparative study analysing women's childbirth satisfaction and obstetric outcomes across two different models of maternity care.

Diaz, T. F., \& Paltrow, L. M. (2010). Birth justice as reproductive justice. .

Direccion General de Salud Pública . (2011). Estrategia para la atención del parto. Servicio Cantabro de Salud, 10.

Establecimientos de Salud Amigos de la Madre y del Niño ESAMyN. (2018, Mayo 2). Retrieved from http://www.salud.gob.ec/establecimientos-de-salud-amigos-de-la-madre-ydel-ninoesamyn/

Fernández, M. (2017). Atención prenatal, experiencia positiva del embarazo. . Matronas Prof. , 48-51.

Freyermunth, G. (2013). Género, etnia y generación, factores constitutivos del riesgo durante la maternidad. CIESAS/ Porrua, 30-40.

Freyermuth, G., \& Sesia, P. (2009). La muerte materna - Acciones y estrategias hacia una meternidad segura. Mexico: Centro de Investigaciones y Estudios Superiores en Antropología Social.

García, E., Guillén, D., \& Acevedo, A. (2010). Humanismo durante la atención del parto. CONAMED, 152-154.

Goberna, P. A., Banús, M., Linares, S., \& Salas, D. (2008). Tecnología y humanización en la asistencia al nacimiento. Matronas Prof., 5-10.

Hodnett, E. (2006). Continuity of caregivers for care during pregnancy and childbirth. Syst Rev., 2.

Iglesias, C., \& Alli, S. (2009). Parto y nacimiento humanizado: evaluación de una vía clínica basada en la evidencia. Matronas Profesion, 5-11.

Lais, J., \& Gilbertg, E. (2012). Caminos hacia una maternidad diferente: un estudio sobre el parto vertical. Revista electronica, 20-25.

Marsden, W. (2016). El cuidado maternal en España 2016: La necesidad de humanizar el parto. Medicina Naturista, 12-13.

Martínez, G. J. (2011). Implantación del nuevo modelo de atención al parto. Enferm Ref., 65-71. 


\section{Parto humanizado como estrategia de salud pública en atención primaria de}

Vol. 2, núm. 3., (2018)

Mariela Gissela Macías Intriago; Ivonne Jasmina Tacoamán Acurio; Luis Alberto Giler Saltos; William Mauricio Quimis Guerrido; María Lourdes Choez Reyes; Melissa Mercedes Álvarez

Lara

Martínez, J., \& Delgado, M. (2014). Contribución de la educación maternal a la salud materno infantil. Matronas Prof., 137-141.

Ministerio de Salud de Brasil. (2000). Humnizacion del parto. Portaria/ GM, 569.

Ministerio de Salud Pública. (2017, 12). Por un parto Culturalmente Adecuado. Retrieved from http://www.care.org.ec/wpcontent/ion-Parto-Culturalmente-Adecuado1.pdf

Ministerio de Sanidad y Consumo. (2007). Estrategia de atención al parto normal en el Sistema Nacional de Salud. Madrid: Ministerio de Sanidad y Consumo.

Misago, K., \& Kendall, C. (2001). From culture of dehumanization of childbirth to childbirth as a transformative experience: changes in five municipalities in north-east Brazil. . Int. J Gynaecol Obstet, 67-72.

Organización Mundial de la Salud (OMS). (1985). Appropiate technology . Lancet, 436-437.

Organización Mundial de la Salud. (1996). Cuidados en el parto normal: Una guía práctica.

Organización Mundial de la Salud. (2016). Recomendaciones de la OMS sobre atención prenatal para una experiencia positiva del embarazo.

Rodríguez, B. M. (2013). ). Ideologías y Prácticas de Género en la Atención Sanitaria del Embarazo, Parto y Puerperio: El caso del Área 12 de la Comunidad de Madrid. Madrid: Tesis Doctoral.

Secretaría Nacional de Planificación. (2007). II Informe Nacional de los objetivos de desarrollo del milenio.

Sistema Nacional de Salud CONASA. (2018, Abril 25). Guía Técnica para la Atención del Parto Culturalmente adecuado. Retrieved from https://www.cappalatinoamerica.com/

Wagner, M. (2001). Fish can't see water: the need to humanize birth. Int J Gynaecol Obstet, 2537.

Zavala, F. (2018, 05 13). Violencia obstetricas. Retrieved from https://informe.gire.org.mx/caps/cap4 